\title{
Diálogos sobre Ciencias Aeronáuticas en Honduras
}

Alex Matamoros

\section{Resumen}

Una de las primeras actividades del Departamento de Ciencias Aeronáuticas (DCA), luego de su conformación, ha sido la presentación de conferencias dirigidas a la población hondureña en general y al gremio aeronáutico en especial. Durante los años 2013 y 2014 se han desarrollado diez y siete conferencias distribuidas en tres espacios de divulgación: 1) un proyecto de vinculación Universidad-Sociedad del DCA denominado "Ciclo de conferencias sobre Aeronáutica Civil en Honduras", 2) los congresos de investigación científica que desarrolla cada año la Dirección de Investigación Científica y Posgrado (DICYP) y, 3) las Jornadas Científicas de la Facultad de Ciencias Espaciales (FACES). El presente artículo hace un breve recorrido por los temas tratados, los conferencistas y el público participante para finalizar proponiendo una serie de conclusiones desde las cuales se pueden mejorar estas prácticas de divulgación científica.

Palabras claves: Conferencias, divulgación científica, población hondureña, gremio aeronáutico, diálogo

\section{Abstract}

One of the first activities of the Department of Aeronautical Sciences (DAC) after its formation has been presenting conferences to the Honduran aerial general population. During the years 2013 and 2014 were developed seventeen conferences on three areas of disclosure: 1) a project linking University-DAC and society called "Cycle of Conferences on Civil Aviation in Honduras", 2) the Congresses of Scientific Research which develops each year the Direccion de Investigacion Cientifica y Posgrado (DICYP) and 3) the Scientific Conferences of the Faculty of Spatial Sciences. This article makes a brief tour of the topics, speakers and participant to finish proposing a series of conclusions from which we can improve practices of scientific disclosure.

Keywords: Conferences, science, Honduran population, aeronautical group, dialogue 
Alex Matamoros, (algemaca@hotmail.com), Departamento de Ciencias Aeronáuticas, Facultad de Ciencias Espaciales, Universidad Nacional Autónoma de Honduras - UNAH 


\section{A manera de introducción}

El nacimiento del Departamento de Ciencias Aeronáuticas es el fruto de un diálogo de la UNAH por medio de la Facultad de Ciencias Espaciales con otros actores vinculados al campo de las Ciencias Aeronáuticas, algunos de ellos del ámbito nacional otros, con proyección internacional. En el primer grupo se pueden mencionar la Asociación para el desarrollo de la Aviación y la Educación en Honduras (ADAEH), la Agencia Hondureña de Aeronáutica Civil (AHAC), antes Dirección General de Aeronáutica Civil (DGAC), la Concesionaria Interairport, y la Corporación Centroamericana de Servicios Aeronáuticos (COCESNA), entre otras; en el segundo grupo se pueden incluir Universidades con experiencia en el campo aeronáutico con las que se han sostenido contacto y con las que se tienen en proceso la firma de convenios de cooperación, como ha sido el caso de la Universidad Tecnológica de Panamá, la Universidad Politécnica de Madrid y el Vaughn College de New York. Además se ha establecido comunicación con profesionales independientes que han brindado valiosas opiniones para el mejoramiento del desempeño del Departamento de Ciencias Aeronáuticas.

Una forma de aprovechar esta relación con la comunidad aeronáutica han sido las diez y siete conferencias impartidas desde el Departamento de Ciencias Aeronáuticas aprovechando al menos tres ámbitos: el proyecto de vinculación denominado Ciclo de Conferencias sobre Ciencias Aeronáuticas, el Congreso de Investigación Científica de la UNAH auspiciado por la Dirección de Investigación Científica y Posgrado que se realiza una vez por Año y la Jornada Científica de la Facultad de Ciencias Espaciales. Hasta la fecha, se han desarrollado tres ciclos de conferencias con ocho conferencias presentadas; se divulgaron tres temas de investigación en el Congreso de investigación de UNAH y se han expuesto cinco proyectos de investigación desarrollados o en desarrollo por parte de la Unidad de Gestión de la Investigación del Departamento de Ciencias Aeronáuticas (DCA) en la Sexta y Séptima Jornada Científica desarrolladas por la Facultad de Ciencias Espaciales de la UNAH.

\section{Finalidad de las conferencias presentadas}

Como se explicaba para el primer ciclo de conferencias la finalidad de estas actividades de divulgación y diálogo científico ha sido la de dar a conocer al público en general y especialmente entre los profesionales y técnicos interesados o vinculados con las ciencias relacionadas con la aeronáutica civil, las temáticas del campo con mayor relevancia en el contexto actual. 
Con las conferencias presentadas se ha logrado un acercamiento por parte de la UNAH a algunas de las principales temáticas del campo de la aeronáutica desde una perspectiva civil. Un momento importante de este relacionamiento se dio con la visita que se tuvo de la Universidad Tecnológica de Panamá, cuando el Dr. Víctor Sánchez nos expuso la experiencia de esta casa de estudios en el establecimiento de un grupo de carreras aeronáuticas dentro de la Facultad de Ingeniería Mecánica (Ver Cuadro 1); en esta ocasión se llamó la atención sobre la importancia de hacer un abordaje desde la Academia que trascienda y complemente el protagonismo de la industria aeronáutica como actor principal, en tal sentido se alabó el hecho de que la UNAH estuviera incursionando en el campo con esta finalidad y capitalizando la larga experiencia del País en la aeronáutica militar y civil. Se pudo ver que en su caso la UTP, ha contado para el desarrollo de la ciencia aeronáutica, con dos apoyos importantes: la Aerolínea COPA, a quienes proveen de profesionales y técnicos aeronáuticos y el Tecnologícal Institute of Florida que le ha proveído de docentes para las asignaturas propiamente aeronáuticas y le ha asesorado el proceso. De todas formas se explicó la importancia de salvaguardar en todo momento la independencia de la Academia con respecto a la industria para no ser absorbida por los criterios del mercado, así como la necesidad de conformar los propios cuadros de docentes para no depender en este aspecto del apoyo externo al País. El desarrollo de esta conferencia que además estuvo acompañada de un intercambio con la Decanatura de la Facultad de Ciencias Espaciales y una visita a la Vicerrectoría de Relaciones Internacionales, se dio en el momento en que el DCA estaba conformando su oferta académica con un Diplomado en Gestión de Sistemas Aeroportuarios y una Licenciatura en Ciencias Aeronáuticas, misma que ahora tiene por nombre Licenciatura en Operaciones Aeronáuticas.

\section{El Ciclo de conferencias sobre Aeronáutica Civil en Honduras}

Como hemos repetido en distintas reuniones con la Decanatura de la FACES, en el momento presente el Departamento de Ciencias Aeronáuticos sólo puede crecer para alcanzar los fines para los cuales fue creado, puesto que se constata en el País una demanda importante de profesionales aeronáuticos que cuenten con grado universitario. Este acierto ha quedado demostrado en el Diagnóstico para la creación de la Licenciatura en Operaciones Aeronáuticas que se encuentra en proceso de aprobación. Pero además ha sido ratificado en las siete conferencias ofrecidas a la comunidad aeronáutica nacional (Cuadro 1), las cuales dan cuenta de una evolución en el conocimiento del campo indagando una amplia diversidad temática que incluye aspectos como la descripción de los principales actores invo- 
lucrados en el campo de la aeronáutica en Honduras, la relación de la aeronáutica con el desarrollo del País, la centralidad del factor humano para la seguridad en las operaciones aeronáuticas, la interacción entre las personas y los sistemas computarizados para la seguridad en las aeronaves, la evolución del derecho aeronáutico a partir del Convenio de Chicago de 1944, un encuadre del rol que corresponde a la Universidad como parte de una nueva comunidad aeronáutica en Honduras, los componentes y la planificación de la seguridad en las operaciones aeronáuticas en los aeropuertos, los procesos de certificación de los operadores aéreos y las ventajas que ofrece a una empresa el contar con una certificación, el conocimiento de algunos de los manuales con los que se debe cumplir para concluir con éxito un proceso de certificación, el proceso de transformación que está viviendo la Autoridad Aeronáutica actualmente para hacerse cargo de su labor normativa, por mencionar algunos de los temas tratados. 
Cuadro 1: Resumen de eventos del Ciclo de conferencias sobre Aeronáutica Civil en Honduras

\begin{tabular}{|c|c|c|}
\hline Conferencia & Sinopsis & Conferencista 2. \\
\hline $\begin{array}{l}\text { 1. Ciencias } \\
\text { Aeronáuticas en } \\
\text { Honduras. Un } \\
\text { panorama general, } \\
9 \text { de mayo de } 2013\end{array}$ & $\begin{array}{l}\text { Ofrece una idea de conjunto del estado actual de } \\
\text { la aeronáutica civil en Honduras, la terminología } \\
\text { que se maneja en este campo, los principales } \\
\text { actores en la materia, los cambios que han } \\
\text { caracterizado el campo, su relación con el } \\
\text { desarrollo del país, los recursos con los que } \\
\text { debería contar, entre otros tópicos. }\end{array}$ & $\begin{array}{l}\text { J urgen Hesse, Licenciado en Ciencias } \\
\text { Aeronaúticas, Piloto de helicópteros, } \\
\text { Docente en la Universidad de la } \\
\text { Defensa. }\end{array}$ \\
\hline $\begin{array}{l}\text { 2. Conferencia: EI } \\
\text { factor humano en } \\
\text { la aeronáutica, } 16 \\
\text { de mayo de } 2013 .\end{array}$ & $\begin{array}{l}\text { Describió los factores que intervienen para hacer } \\
\text { posible la aeronavegación, haciendo énfasis en la } \\
\text { centralidad que tiene la persona humana, con la } \\
\text { cual interactúan el soporte lógico, el equipo y el } \\
\text { ambiente. }\end{array}$ & $\begin{array}{l}\text { Gerardo Inestroza, Licenciado en } \\
\text { Ciencias Aeronáuticas, Piloto de ala fija } \\
\text { y ala rotatoria, instructor de vuelo. }\end{array}$ \\
\hline $\begin{array}{l}\text { 3. Legislación } \\
\text { aeronáutica. Piedra } \\
\text { Angular de la } \\
\text { Aviación Civil, } 23 \\
\text { de mayo de } 2013\end{array}$ & $\begin{array}{l}\text { Propone que el Derecho Aeronáutico es una rama } \\
\text { nueva del Derecho que encuentra sus fuentes en } \\
\text { el Derecho Civil, Comercial, Administrativo, } \\
\text { Laboral, Marítimo, Internacional y comparado } \\
\text { Público y Privado, a los cuales ha enriquecido. } \\
\text { Hace un breve recorrido histórico de las } \\
\text { concepciones del derecho aeronáutico y un repaso } \\
\text { de sus concepciones en distintos países. }\end{array}$ & $\begin{array}{l}\text { Pavel Andrey Espinal, Sub Director } \\
\text { de la Dirección de Aeronáutica Civil, } \\
\text { Miembro Fundador de ADAEH, } \\
\text { integrante del equipo interdisciplinario } \\
\text { para conformar el Departamento de } \\
\text { Ciencias Aeronáuticas de la UNAH. }\end{array}$ \\
\hline $\begin{array}{l}\text { 4. Desarrollo del } \\
\text { campo aeronáutico } \\
\text { en la Academia: el } \\
\text { caso de la } \\
\text { Universidad } \\
\text { Tecnológica de } \\
\text { Panamá. } 21 \text { de julio } \\
\text { de } 2013 . \\
\end{array}$ & $\begin{array}{l}\text { Se señala la necesidad de abordar el tema en la } \\
\text { perspectiva de la Academia, aun en el caso de } \\
\text { Panamá, en donde se está formando para } \\
\text { responder a la demanda de COPA que representa } \\
\text { para ellos el mayor mercado para sus egresados. } \\
\text { Sigue siendo para ellos un desafío la formación de } \\
\text { los propios docentes para el campo aeronáutico. }\end{array}$ & $\begin{array}{l}\text { Víctor Sánchez, Coordinador del } \\
\text { Departamento de Ciencias } \\
\text { Aeronáuticas de la Facultad de } \\
\text { Ingeniería de la Universidad } \\
\text { Tecnológica de Panamá. }\end{array}$ \\
\hline $\begin{array}{l}\text { 5. Sistema de Gestión } \\
\text { de la Seguridad } \\
\text { Operacional en } \\
\text { Aeródromos } \\
\text { (SIGESOA), } 7 \text { de } \\
\text { noviembre de } 2013\end{array}$ & $\begin{array}{l}\text { La seguridad operacional es la aplicación del } \\
\text { anexo } 19 \text { de la OACI y la importancia de su } \\
\text { aplicación deriva de la latencia de amenazas de } \\
\text { incidentes y accidentes en el ambiente de trabajo } \\
\text { de un aeropuerto. La mitigación de peligros } \\
\text { requiere el control de todos los elementos que } \\
\text { interactúan en el ambiente aeroportuario. La } \\
\text { atención al SIGESOA requiere de planificación e } \\
\text { inversión, sin descuidar la rentabilidad del rubro } \\
\text { aeronáutico. }\end{array}$ & $\begin{array}{l}\text { J orge Gutiérrez, Gerente de } \\
\text { Operaciones de Interairport, Dirigió la } \\
\text { reestructuración del Aeropuerto } \\
\text { Toncontín desde el inicio de su } \\
\text { concesión. }\end{array}$ \\
\hline $\begin{array}{l}\text { 6. Certificación de } \\
\text { Operadores } \\
\text { Aéreos, } 14 \text { de } \\
\text { noviembre de } 2013\end{array}$ & $\begin{array}{l}\text { Se presentó el proceso completo en sus cinco } \\
\text { fases de pre solicitud, solicitud formal, evaluación } \\
\text { documental, evaluación técnica y entrega de la } \\
\text { certificación. El proceso cumple con una serie de } \\
\text { manuales entre ellos el Manual General de } \\
\text { Operaciones (MGO), el Manual de Control de } \\
\text { Mantenimiento (MCM), la Lista de Equipo Mínimo } \\
\text { para despacho de la aeronave (M.E.L.) y el } \\
\text { Programa de Mantenimiento de la Aeronave } \\
\text { (PMA). Se concluye con algunos de los beneficios } \\
\text { que ofrecen las certificaciones. }\end{array}$ & $\begin{array}{l}\text { Iván Betancourt, Ingeniero Mecánico. } \\
\text { Especialista en Ingeniería y } \\
\text { mantenimiento aeronáutico, Consultor } \\
\text { para procesos de certificación de } \\
\text { operadores aéreos, Asesor Técnico en } \\
\text { Sistema de Calidad Aeronáutico. } \\
\text { Docente del Departamento de Ciencias } \\
\text { Aeronáuticas }\end{array}$ \\
\hline
\end{tabular}




\begin{tabular}{|c|c|c|}
\hline $\begin{array}{l}\text { 7. Metas de la DGAC } \\
\text { para el período 2014- } \\
\mathbf{2 0 1 8}, 15 \text { de agosto de } \\
\mathbf{2 0 1 4}\end{array}$ & $\begin{array}{l}\text { Se presenta el proceso de reconfiguración que } \\
\text { está viviendo la Autoridad Aeronáutica } \\
\text { hondureña en su paso de Dirección General de } \\
\text { Aeronáutica Civil a Agencia Hondureña de } \\
\text { Aviación Civil. La reforma propuesta contribuirá } \\
\text { a la priorización del personal técnico sobre al } \\
\text { administrativo, la independización de las } \\
\text { decisiones y el mejor cumplimiento de la } \\
\text { Autoridad Aeronáutica de los Anexos OACl. } \\
\text { Una meta en el corto y mediano plazo es la } \\
\text { categorización del País por parte de la FAA. }\end{array}$ & $\begin{array}{l}\text { Guido Lucio Venegas, Ex Piloto de la } \\
\text { desaparecida línea aérea SAHSA, Jefe } \\
\text { de Estándares de Vuelo de la DGAC, } \\
\text { con experiencia en distintos cargos en } \\
\text { esta Dirección. }\end{array}$ \\
\hline $\begin{array}{l}\text { 8. La } \\
\text { Municipalización } \\
\text { de los } \\
\text { Aeropuertos en } \\
\text { Honduras como } \\
\text { clave del } \\
\text { desarrollo } \\
\text { regional, } 15 \text { de } \\
\text { agosto de } 2014\end{array}$ & $\begin{array}{l}\text { Este panel expuso las posibilidades que tiene } \\
\text { Honduras para potenciar su desarrollo, } \\
\text { económico, social y turístico con el apoyo del } \\
\text { transporte aéreo. Entre las condiciones } \\
\text { necesarias para que esto sea posible se } \\
\text { mencionan una planificación estratégica de las } \\
\text { movilizaciones dentro del territorio y desde y } \\
\text { hacia afuera de éste, la priorización de las } \\
\text { zonas con mayor potencial para la movilización } \\
\text { de cargas y personas, el trabajo conjunto entre } \\
\text { la inversión privada y el Estado, el } \\
\text { acercamiento del transporte aéreo a la } \\
\text { cotidianeidad, la visibilización de las muchas } \\
\text { funciones que cumple un aeropuerto y el bench } \\
\text { marking aeroportuario, entre otros. }\end{array}$ & $\begin{array}{l}\text { Vivian Cárdenas, Msc en Economía } \\
\text { aplicada al Turismo, Consultora en } \\
\text { temas de desarrollo sostenible. } \\
\text { Isaí Fearon, Lic. en Administración de } \\
\text { Aeropuertos, Master en Dirección } \\
\text { Empresarial, Piloto Aviador. } \\
\text { Gerardo Inestroza, Lic. en Ccs. } \\
\text { Aeronáuticas, Piloto, con experiencia } \\
\text { de más de quince años como instructor } \\
\text { de vuelo. }\end{array}$ \\
\hline
\end{tabular}

Fuente: elaboración propia en base a las memorias elaboradas

Los ciclos de conferencia han permitido un contacto vital con el acontecer aeronáutico nacional y han abierto la posibilidad de proponerse nuevos temas para nuevas conferencias, plantear nuevas problemáticas para investigar y establecer contactos para continuar conquistando espacios en la comunidad aeronáutica. Sin caer en la autocomplacencia se puede decir que las conferencias han cumplido con su razón de ser, puesto que han coadyuvado a mantener abierto un espacio de comunicación activo al que se ha recurrido desde el DCA en varias ocasiones para distintas finalidades.

\section{El congreso de Investigación Científica de la UNAH}

Otro espacio de divulgación han sido las participaciones en la Congresos de investigación científica de la UNAH de la Dirección de Investigación Científica y Posgrados (DICYP). Las exposiciones principales que ha ofrecido el Departamento han sido para dar a conocer a la comunidad universitaria dos proyectos de investigación que se han culminado como parte de la carga académica de investigación del Departamento se trata de la conferencia "Tendencias a nivel nacional de la demanda de profesionales aeronáuticos universitarios" y el panel de expertos "La especialización técnica en control de tránsito aéreo en una licenciatura en Ciencias 
Aeronáuticas" (Ver Cuadro 2). La conferencia se presentó en el Año 2013 cuando se estaba finalizando la primera versión del Diagnóstico para la Licenciatura en Operaciones Aeronáuticas; el panel realizado en 2014 aportó algunas de las ideas incorporadas al proyecto de investigación con el que se dio contenido y orientación a la parte correspondiente al Técnico Controlador de Tránsito Aéreo que se podría incluir dentro de la Licenciatura antes mencionada. Adicionalmente en este espacio se presentó uno de los trabajos realizados por los estudiantes del Diplomado en Gestión de Sistemas Aeroportuarios que abrió un debate muy necesario para la aeronáutica hondureña, se hace referencia a la conferencia "El Consejo Aeronáutico Nacional (CAN): análisis de su situación actual en el marco institucional de la aeronáutica civil hondureña".

Al aprovechar este espacio abierto por la DICYP, se ha fortalecido el proceso de conformación de la oferta académica del Departamento, incorporando criterios de pertinencia provenientes de expertos del campo aeronáutico integrados con los principios orientadores de la Academia que se derivan del Modelo Educativo de la UNAH. Además, se ha abierto un espacio de divulgación de los temas de investigación e innovación dentro de la comunidad científica universitaria, al grado que la presentación de trabajos en este Congreso es ya parte de la vida académica del DCA. 
Cuadro 2: Resumen de participaciones en el Congreso de Investigación Científica de la DICYP-UNAH

\begin{tabular}{|c|c|c|}
\hline Conferencia & Sinopsis & Conferencista \\
\hline $\begin{array}{l}\text { 1. Tendencias a } \\
\text { nivel nacional } \\
\text { de la demanda } \\
\text { de } \\
\text { profesionales } \\
\text { aeronáuticos } \\
\text { universitarios, } \\
7 \text { de agosto de } \\
2013 \text {. }\end{array}$ & $\begin{array}{l}\text { Se explica la importancia de contar en Honduras con } \\
\text { un desarrollo de la Aeronáutica Civil. La investigación } \\
\text { propone como problema de referencia la falta en } \\
\text { Honduras de personal técnico aeronáutico con los } \\
\text { conocimientos propios del nivel universitario para una } \\
\text { buena gestión del campo aeronáutico. Esta carencia ha } \\
\text { contribuido a que el país se haya incorporado } \\
\text { débilmente a las tendencias regionales e } \\
\text { internacionales aeronáuticas. Considerando que la } \\
\text { aeronáutica es por su naturaleza internacional, se } \\
\text { exponen las tendencias internacionales de los } \\
\text { currículos de formación y los respectivos perfiles de } \\
\text { salida. }\end{array}$ & $\begin{array}{l}\text { Omri Alberto Amaya, Lic. En } \\
\text { Ciencias Aeronáuticas, Ingeniero } \\
\text { Industrial, Piloto Comercial, Jefe } \\
\text { del Departamento de Ciencias } \\
\text { Aeronáuticas FACES/UNAH. }\end{array}$ \\
\hline $\begin{array}{l}\text { 2. El Consejo } \\
\text { Aeronáutico } \\
\text { Nacional (CAN): } \\
\text { análisis de su } \\
\text { situación actual } \\
\text { en el marco } \\
\text { institucional de } \\
\text { la aeronáutica } \\
\text { civil } \\
\text { hondureña", } \\
4 \text { de agosto de } \\
2014 .\end{array}$ & $\begin{array}{l}\text { La Ley de Aeronáutica Civil obliga a la conformación } \\
\text { del CAN; sin embargo es este un imperativo que el } \\
\text { País no ha atendido. En los países en donde se ha } \\
\text { conformado este equipo, adquiere características de } \\
\text { interdisciplinariedad y elabora las políticas, estrategias } \\
\text { y objetivos que orientan la aeronáutica civil; se ilustra } \\
\text { este hecho presentando los casos de Chile, Ecuador y } \\
\text { República Dominicana. Para optimizar su } \\
\text { funcionamiento el CAN tiene que dejar de ser un } \\
\text { órgano consultivo para convertirse en el órgano que } \\
\text { rectore el desarrollo de la aeronáutica en Honduras. }\end{array}$ & $\begin{array}{l}\text { Ana Lucía Ulloa, Lic. En } \\
\text { Mercadotecnia, Master en } \\
\text { Gestión de Proyectos, } \\
\text { Diplomada en Gestión de } \\
\text { Sistemas Aeroportuarios. }\end{array}$ \\
\hline $\begin{array}{l}\text { 3. La } \\
\text { especialización } \\
\text { técnica en } \\
\text { control de } \\
\text { tránsito aéreo } \\
\text { en una } \\
\text { licenciatura en } \\
\text { Ciencias } \\
\text { Aeronáuticas, } \\
5 \text { de agosto de } \\
2014 .\end{array}$ & $\begin{array}{l}\text { Fue un panel de expertos que contó con la } \\
\text { participación de COCESNA, la DGAC y de la UNAH. } \\
\text { Por su parte COCESNA relató su experiencia en el } \\
\text { campo a nivel centroamericano, resaltando la } \\
\text { rigurosidad de la formación recibida en la institución } \\
\text { para el desarrollo de sus labores profesionales. Por su } \\
\text { parte DGAC expone que esta institución asume su } \\
\text { responsabilidad como Autoridad Aeronáutica en cuanto } \\
\text { al tránsito aéreo por medio de la Dirección de Tránsito } \\
\text { Aéreo; se explica que al examinar el concepto de } \\
\text { tránsito aéreo se descubre una gran amplitud de } \\
\text { actividades incluidas que van desde alertas hasta } \\
\text { control sobre vuelos militares, pasando por } \\
\text { comunicaciones y todo lo relacionado con vuelos } \\
\text { civiles; el principal desafío de la DGAC sigue siendo la } \\
\text { falta de personal técnico y presupuesto para atender } \\
\text { sus funciones. Por último el DCA de FACES-UNAH, } \\
\text { enfatiza en la importancia de contar con Controladores } \\
\text { con formación universitaria para fortalecer sus } \\
\text { competencias técnicas. }\end{array}$ & $\begin{array}{l}\text { Víctor Manuel Andrade, } \\
\text { Controlador de Tránsito Aéreo } \\
\text { de COCESNA con } 25 \text { años de } \\
\text { experiencia. } \\
\text { Heriberto Sierra, Jefe } \\
\text { Departamento de Navegación } \\
\text { Aérea de AHAC. } \\
\text { Alberto Colindres, Lic. En } \\
\text { Ciencias Aeronáuticas, Piloto } \\
\text { Comercial, Controlador Aéreo de } \\
\text { combate. }\end{array}$ \\
\hline
\end{tabular}

Fuente: elaboración propia en base a Memorias de las conferencias presentadas. 


\section{Jornadas Científicas de la FACES}

El compartir los proyectos de investigación dentro de la Facultad de Ciencias Espaciales ha sido también un estímulo importante para mejorar los enfoques y las metodologías propuestas y para descubrir campos en los cuales podríamos establecer sinergias con el trabajo de los demás departamentos. Las conferencias presentadas en este espacio propio de la Facultad han sido básicamente para dar a conocer los proyectos de investigación que luego han sido enviados a la DICYP para su revisión (Ver Cuadro 3). Cabe mencionar que dos de los proyectos presentados en este espacio de divulgación interna, son ya artículos que en este momento están en revisión por pares externos para ser incluidos en la Revista Ciencias Espaciales, se trata de los proyectos "Diseño para establecer el currículo de Controlador de Tránsito Aéreo dentro de la Licenciatura en ciencias aeronáuticas" y "Contenidos de una asignatura optativa del Departamento de Ciencias Aeronáuticas-Introducción a las Aeronaves". 
Cuadro 3: Resumen de conferencias de las Jornadas Científicas de FACES

\begin{tabular}{|l|}
\hline Conferencia \\
\hline 1. Diseño para \\
establecer la currículo \\
de Controlador de \\
Tránsito Aéreo dentro \\
de la Licenciatura en \\
ciencias aeronáuticas, \\
17 de diciembre de \\
2013
\end{tabular}

2. Alternativa para la reactivación de la industria Aeroespacial de Honduras, 17 de diciembre de 2013

\section{Sinopsis}

Se presentó el proyecto de investigación que

propuso preliminarmente cuatro asignaturas para la

formación de Controladores: ATC 1 incluyendo

movimiento de aeronaves, vehículos y personas en

el área de maniobras; ATC 2, autorización de

despegue de aeronaves; ATC 3 Autorización de

aterrizajes de aeronaves; ATC 4 incluyendo

suministro de información meteorológica, NOTAM,

conciencia situacional, manejo de situaciones de

amenaza y error. Se expusieron los requisitos

requeridos en Honduras para ser Controlador.

Esta conferencia tiene el nombre de una Tesis de posgrado defendida en una Universidad hondureña.

El propósito de este estudio es investigar las deficiencias de la industria aeroespacial, mapear y caracterizar las empresas relacionadas con esta industria, para poder definir cómo se podría desarrollar este campo teniendo en cuenta los objetivos y capacidades de la DGAC como autoridad reguladora.

3. Contenidos de una asignatura optativa del Departamento de Ciencias

AeronáuticasIntroducción a las Aeronaves, $17 \mathrm{de}$ diciembre de 2013

4. Necesidades de formación del personal de la Industria Aeronáutica en el Aeropuerto Toncontín de Tegucigalpa, 17 de diciembre de 2013

5. Orientaciones para el diseño de la

Estructura de la Escuela de Ciencias Aeronáuticas de la Facultad de Ciencias Espaciales de la Universidad Nacional de Autónoma de Honduras, 17 de diciembre de 2013
Esta investigación sirvió de base para diseñar la primera asignatura ofrecida por el DCA a la comunidad universitaria. Sus contenidos muestran los conceptos básicos fundamentales de las aeronaves: sus generalidades, sus partes, descripción y tipos. Principios básicos de aerodinámica, comportamiento en vuelo $\mathrm{e}$ instrumentos de la aeronave, generalidades de los motores en aviación, sistemas de la aeronave, entre otros temas.

Este proyecto de investigación buscaba identificar qué modalidades de educación están siendo implementadas para la capacitación del personal que actualmente labora en la industria aeronáutica en el Aeropuerto Internacional del Toncontín, para luego deducir qué criterios deberían orientar los contenidos utilizados o por utilizar para su formación.

Esta conferencia presentó los lineamientos que debe seguir la conformación de la Escuela de Ciencias Aeronáuticas dentro de la nueva estructura de la Facultad de Ciencias Espaciales. Se contará con dos Departamentos: uno de Ciencias

Aeronáuticas y otro de Aviación; la Escuela será la unidad académica encargada de liderar el desarrollo de las Ciencias Espaciales en Honduras, en el campo de la Aeronáutica, mediante la implementación de la investigación, la vinculación universidad-sociedad y la gestión académica. a.
Conferencista

Alberto Colindres, Lic. En

Ciencias Aeronáuticas,

Piloto Comercial,

Controlador Aéreo de

combate, Catedrático de la

Universidad de la Defensa.

Isaí Fiaron, Lic. en

Administración de

Aeropuertos, Master en

Dirección Empresarial,

Piloto Aviador

Iván B etancourt, Ingeniero Mecanico. Especialista en Ingeniería y mantenimiento aeronáutico, Consultor para procesos de certificación de operadores aéreos, Asesor Técnico en Sistema de Calidad Aeronáutico.

Docente del Departamento de Ciencias Aeronáuticas

Alex Matamoros, Master en Calidad y Equidad de la Educación, Diplomado en Gestión de Sistemas Aeroportuarios, Docente del Departamento de Ciencias Aeronáuticas.

Omri Alberto Amaya, Lic. En Ciencias Aeronáuticas, Ingeniero Industrial, Piloto Comercial, Jefe del Departamento de Ciencias Aeronáuticas FACES/UNAH. 


\begin{tabular}{|l|l|l|}
\hline 6.Cuerpo de & Este artículo presenta las partes principales de un & Alberto Colindres, Lic. En \\
Contenido para la & trabajo preparado para adaptar un Técnico en & Ciencias Aeronáuticas, \\
Formación de & Controlador de Tránsito Aéreo (ATC) a la & Piloto Comercial, \\
Controlador de & Licenciatura en Ciencias Aeronáuticas que & Controlador Aéreo de \\
Tránsito Aéreo en el & actualmente (diciembre de 2014) se encuentra en & combate, Catedrático de la \\
ámbito de las & proceso de aprobación por parte de la Autoridades & Universidad de la Defensa. \\
Ciencias Aeronáuticas & Académicas de la UNAH. Los principales resultados & \\
en Honduras, & presentados son 1. El espectro de posibilidades de \\
12 de diciembre de & empleo que tiene el control de tránsito aéreo en la \\
2014. & Agencia Hondureña de Aeronáutica Civil (AHAC); 2. \\
& La composición de la carrera de Técnico \\
& Aeronáutico ATC u otras carreras similares en & \\
& distintos Centros de Formación Superior, 3. Las & \\
& opiniones de distintos profesionales aeronáuticos & \\
& sobre la formación y el desempeño de los ATC en & \\
& Honduras, 4. La composición de las asignaturas que & \\
& incluiría un Técnico ATC propuesto desde el & \\
& Departamento de Ciencias Aeronáuticas de FACES. & \\
\hline
\end{tabular}

Fuente: Elaboración propia en base a las Memorias de las Jornadas Científicas

FACES de 2013 y 2014.

\section{Los protagonistas: Conferencistas y participantes}

El campo de las Ciencias Aeronáuticas Civiles en Honduras se encuentra en proceso de conformación y en ese sentido es de esperar, como lo muestran las conferencias presentadas, que esté siendo analizado por profesionales propios del campo aeronáutico y por otros que están aportando desde su propia formación profesional universitaria. En el primer grupo destacan aquellos que han egresado de la Academia militar, muchos de los cuales están trabajando en el ámbito civil y muestran algún interés por continuar profundizando conocimientos de la aeronáutica civil o bien devienen obligados a hacerlo al estar al frente de puestos importantes dentro de la AHAC; el segundo grupo es de quienes más se ha obtenido colaboración y se encuentran entre ellos Abogados, Ingenieros y Licenciados.

Una de las reglas de selección de conferencistas ha sido la de ostentar un grado universitario, sin embargo se obtuvo la colaboración de COCESNA por medio de uno de sus técnicos con una experiencia de más de veinte años que sustituyó con creces el requisito. Se llama la atención sobre este hecho para mostrar que el diálogo abierto ha dado cabida también a los aportes de técnicos sin título universitario que dicho sea de paso conforman la mayor parte de la población empleada en el campo aeronáutico en Honduras y posiblemente en el mundo. 
Resalta el hecho de que una de las diez y siete conferencias reportadas fue internacional y contó con la participación de un representante de una universidad de la Región Centroamericana. Uno de los frutos de este intercambio con la Universidad Tecnológica de Panamá es un convenio específico de colaboración que ahora mismo está en proceso de firma.

En términos generales se puede decir que por tratarse de profesionales y técnicos activos en el campo, los conferencistas estuvieron en grado de aportar criterios basados en conocimientos actualizados del campo aeronáutico. Además, proveyeron al Departamento de Ciencias Aeronáuticos de temas sobre los cuales merece la pena seguir profundizando en las ciencias aeronáuticas con el montaje de nuevos eventos similares o acometiendo nuevas investigaciones. Adicionalmente por medio de estos expositores en muchos de los casos se pudo acceder a niveles gerenciales de empresas aeronáuticas 0 a personal con poder de decisión en su propia institución. Por último, cabe mencionar que en muchos de los casos los expositores han sido incorporados a los bancos de datos de docentes del DCA con altas posibilidades de convertirse en docentes.

Todas las conferencias se hicieron en las instalaciones de la Facultad de Ciencias Espaciales y contaron con la participación como oyentes de estudiantes de la UNAH, docentes de FACES, alumnos de escuelas de aviación, empleados de la AHAC, estudiantes de la Fuerza Aérea Hondureña y empleados de empresas aeronáuticas; con esta audiencia se llegó los representantes de todas las áreas de la industria y la institucionalidad del Estado relacionados con el ambiente aeronáutico nacional.

\section{Conclusiones}

- Tomando en cuenta el cumplimiento de objetivos, la participación del público, la pertinencia de las conferencias y los conferencistas, la calidad de las intervenciones de los participantes y la presencia institucional, las conferencias presentadas por el Departamento de Ciencias Aeronáuticas se consideran exitosas.

- Los contenidos de las conferencias y los contactos interinstitucionales que se mantuvieron durante su desarrollo ofrecen una base importante para generar información para orientar la generación y el enriquecimiento de la oferta académica del Departamento de Ciencias Aeronáuticas; además nos han proveído de temas nuevos de investigación y de socios potenciales para llevar a cabo estas investigaciones. 
- Las relaciones institucionales generadas en durante las conferencias, como se pudo ver en el caso de la Universidad Tecnológica de Panamá, pueden dar lugar a asocios estratégicos que pueden ser sancionados por medio de convenios marco y específicos.

- La composición del público de las conferencias da la posibilidad de conformar un banco de datos con el cual se pueden y se deben seguir promoviendo todos los eventos públicos programados por el Departamento.

- Con la presentación de los eventos desarrollados el DCA ha establecido una relación orgánica con la comunidad universitaria y con representantes claves del sector aeronáutico nacional; desde las muchas perspectivas que pueden derivar de este relacionamiento se pueden hacer diversos tipos de abordaje sobre los grandes temas actuales de la aeronáutica.

- Muchos de los temas abordados de manera breve durante las conferencias podrían ser retomados en espacios de diálogo y estudio (charlas, jornadas de trabajo, planificaciones interinstitucionales, entrevistas a informantes claves, otros) que propicien intercambios más específicos para investigaciones coordinadas destinadas a convertirse en nuevas conferencias.

- En una siguiente etapa de conferencias cabe la posibilidad de incorporar eventos que contemplen video conferencias para facilitar la participación de los socios de la Facultad de Ciencias Espaciales en materia aeronáutica con sedes en otros países.

\section{Agradecimientos}

Los eventos públicos recensionados en este artículo fueron posibles por la colaboración abierta y generosa de los socios naturales con los que actualmente cuenta el Departamento de Ciencias Aeronáuticas de FACES. A ellos va nuestro agradecimiento y nuestro ofrecimiento para seguir trabajando juntos en el campo de la aeronáutica civil en Honduras que tiene por delante muchos retos a los que sólo se les puede hacer frente desde una labor coordinada. 